\title{
BMJ Open Diagnostic performance and prognostic value of elastography in patients with biliary atresia and after hepatic portoenterostomy: protocol for a systematic review and meta-analysis
}

\author{
Hualin Yan (D) , ${ }^{1}$ Lanxin Du, ${ }^{1}$ Jiaojiao Zhou, ${ }^{1}$ Yan $\mathrm{Li}^{2}$ Jing Lei, ${ }^{1}$ Juxian Liu (D) , \\ Yan Luo ${ }^{1}$
}

To cite: Yan H, Du L, Zhou J, et al. Diagnostic performance and prognostic value of elastography in patients with biliary atresia and after hepatic portoenterostomy: protocol for a systematic review and meta-analysis. BMJ Open 2021;11:e042129. doi:10.1136/ bmjopen-2020-042129

- Prepublication history and additional material for this paper are available online. To view these files, please visit the journal online (http://dx.doi org/10.1136/bmjopen-2020042129).

Received 26 June 2020 Revised 21 January 2021 Accepted 25 January 2021

\section{Check for updates}

(C) Author(s) (or their employer(s)) 2021. Re-use permitted under CC BY-NC. No commercial re-use. See rights and permissions. Published by BMJ.

${ }^{1}$ Department of Medical Ultrasound, West China Hospital, Sichuan University, Chengdu, China

${ }^{2}$ Department of Ultrasound, Peking Union Medical College Hospital, Chinese Academy of Medical Sciences \& Peking Union Medical College, Beijing, China

\section{Correspondence to}

Dr Juxian Liu;

ljxhuaxip1@126.com and

Dr Yan Luo;

luoyanddoc@163.com

\section{ABSTRACT}

Introduction Biliary atresia (BA) is a life-threatening disease with persistent neonatal cholestasis and progressive liver fibrosis. Timely non-invasive diagnosis of BA can result in early hepatic portoenterostomy (HPE) and better prognosis. Quantitative elastography enables the non-invasive measurement of liver stiffness. However, the studies on elastography methods in the diagnosis of BA and the prediction of post-HPE outcomes vary in their results and have small sample sizes. Thus, we propose this systematic review and meta-analysis to obtain comprehensive evidence on the value of elastography in BA.

Methods and analysis We will search the PubMed, Embase and the Cochrane Central Register of Controlled Trials databases for studies evaluating the diagnostic performance of elastography in patients with $B A$ and the prognostic value of postoperative elastography, from inception to 31 December 2020. We plan to use the Quality Assessment of Diagnostic Accuracy Studies-2 list and the Quality In Prognosis Studies tool to assess the risk of bias in the included studies and the study quality. We will evaluate the diagnostic performance of elastography by synthesising the pooled sensitivity, pooled specificity, pooled positive likelihood ratio, pooled negative likelihood ratio, pooled diagnostic $\mathrm{OR}$ and summary receiver operating characteristic curve using Meta-Disc V.1.4. We will evaluate the predictive value of elastography after HPE by synthesising the pooled correlation coefficient and pooled OR of prognostic outcomes using STATA V.14. The funnel plot and Egger's test will be used to evaluate the potential publication bias. Sensitivity analysis will be conducted by examining the estimated effects of individual studies.

Ethics and dissemination As this study is a meta-analysis based on previously published literature, ethical approval is not necessary according to the ethics committee of West China Hospital, Sichuan University. The results of this study will be published in a peer-reviewed journal.

PROSPERO registration number CRD42020162055.

\section{INTRODUCTION}

Biliary atresia (BA) is an inflammatory obliterative cholangiopathy involving intrabiliary and extrabiliary trees that presents as

\section{Strengths and limitations of this study}

- This study will be the first systematic review and meta-analysis to investigate the diagnostic performance and prognostic value of elastography in patients with biliary atresia and after hepatic portoenterostomy.

- We will determine the cut-off value and pooled diagnostic performance for patients with biliary atresia preoperatively.

- We will determine the pooled prognostic outcomes of elastography after hepatic portoenterostomy of patients with biliary atresia.

- We will use the Quality Assessment of Diagnostic Accuracy Studies-2 list to assess the quality of diagnostic studies and the Quality In Prognosis Studies tool to assess the quality of prognostic studies.

- The main limitation is the inevitable heterogeneity of included studies and potential confounding factors.

persistent neonatal cholestasis and progressive liver fibrosis and cirrhosis. Without surgical treatment, end-stage liver disease ensues, and patients die in the first 2 years of life. ${ }^{1}$ Kasai hepatic portoenterostomy (HPE) remains the standard treatment for $\mathrm{BA}$, and early HPE (before 46 days of life) results in a higher rate of survival with native liver and better long-term clinical outcomes. ${ }^{2}$ Intraoperative cholangiography (IOC) and histopathological examination are the gold standards for BA diagnosis. ${ }^{3}$ However, timely non-invasive diagnosis of BA remains challenging in infants with cholestasis caused by various diseases.

Conventional ultrasound (US) is recommended for BA diagnosis. Although it has good diagnostic performance, normal ultrasonography findings do not rule out nonsyndromic BA. ${ }^{3}$ In addition, it is difficult to 
evaluate liver fibrosis and postoperative outcomes based on conventional US. Since patients with BA develop progressive liver fibrosis in early infancy, the assessment of liver stiffness by elastography may facilitate the differential diagnosis and evaluation of postoperative prognosis.

Quantitative elastography enables the non-invasive measurement of liver stiffness through shear wave propagation. The main quantitative elastography techniques include transient elastography (TE), acoustic radiation force impulse (ARFI) and shear wave elastography (SWE). ${ }^{4}$ There are accumulating reports of the application of quantitative elastography methods in the diagnosis of BA and prediction of post-HPE outcomes. ${ }^{5-12}$ However, these studies vary in the diagnostic performance and prognostic value of elastography, and they have small sample sizes. Thus, we will conduct this systematic review and meta-analysis to synthesise the results and obtain comprehensive evidence on the value of elastography in BA.

\section{MATERIAL AND METHODS}

The protocol is in accordance with the Preferred Reporting Items for Systematic Reviews and Meta-Analyses statement. As this is a systematic literature research, ethical approval is waived.

\section{Inclusion and exclusion criteria}

We will include all prospective or retrospective studies that meet the following criteria:

1. Participants: patients with a diagnosis of BA confirmed by surgical exploration, IOC under laparoscopy or liver biopsy.

2. Interventions: Liver and/or spleen stiffness was measured before and/or after HPE by SWE, TE or ARFI.

3. Comparison: Patients without BA as controls.

4. Outcomes:

- The cut-off value and diagnostic performance of the preoperative liver stiffness value measured by SWE, TE or ARFI for BA diagnosis.

- The coefficient of correlation between the preoperative liver stiffness value and the histological METAVIR Score of liver biopsy.

- The coefficients of correlation between the postoperative liver stiffness value (after HPE) and serum total bilirubin and direct bilirubin levels.

- The prognostic ORs associated with the correlations between the postoperative liver and/or spleen stiffness value (after HPE) and the complications of portal hypertension after HPE, including caput medusae, oesophageal varices or variceal bleeding, ascites, splenomegaly and thrombocytopenia.

- The prognostic ORs associated with the correlations between the postoperative liver stiffness value (after HPE) and the need for liver transplantation.

Exclusion criteria:

1. The study did not contain a control group.
2. The patients in the study had congenital diseases other than BA.

\section{Search strategy}

From inception to 31 December 2020, PubMed, Embase and the Cochrane Central Register of Controlled Trials will be searched using the MeSH terms "biliary atresia", "portoenterostomy", "hepatic", "ultrasonography", "diagnostic imaging" and "elasticity imaging technique", and the related keywords "elastography" and "Kasai procedure". The languages will not be limited in our search strategy. The search strategy of PubMed is presented in online supplemental table 1 .

\section{Data abstraction}

HY and LD will independently assess the eligibility of reports from the title and/or abstract. A third reviewer, JL, will join them to resolve any disagreements. Studies that meet the inclusion criteria will be selected for further analysis. For included studies, we will ask for the original data from corresponding authors for diagnosis and prognosis analysis.

\section{Assessment of risk of bias in included studies and study quality}

We plan to use the Quality Assessment of Diagnostic Accuracy Studies-2 (QUADAS-2) list for the quality assessment of diagnostic studies ${ }^{13}$ and the Quality In Prognosis Studies (QUIPS) tool to assess the quality of prognostic studies. ${ }^{14}$ QUADAS-2 includes four domains: patient selection, index test, reference standard and flow and timing. All domains are assessed in terms of risk of bias, and the first three domains are also assessed in terms of concerns regarding applicability. The results of the assessment will be presented as high risk, low risk or unclear risk. ${ }^{13}$ A summarised bar graph of the percentage of studies that have a low, a high or an unclear risk of bias or concerns about applicability for each domain will be presented. The QUIPS includes six domains: study participation, study attrition, prognostic factor measurement, outcome measurement, study confounding factors and statistical analysis and reporting. The results of all the domain assessments will be presented as high risk, moderate risk or low risk. ${ }^{14}$

\section{Assessment of publication bias}

We plan to use the funnel plot and Egger's test to evaluate the potential publication bias by STATA V.14 (StataCorp LP, College Station, Texas, USA). $\mathrm{P}<0.05$ will be considered to indicate significant publication bias.

\section{Assessment of heterogeneity}

The $\chi^{2}$ test will be used to examine heterogeneity in pooling analysis. Heterogeneity is considered to be statistically significant when $\mathrm{p}<0.10$ in these qualitative tests. We plan to use the $I^{2}$ test to estimate the proportion of total variation across studies that is attributable to heterogeneity rather than chance, with values of $25 \%, 50 \%$ and $75 \%$ indicating low, moderate and high inconsistency, respectively. To 
determine the source of heterogeneity, we will conduct a meta-regression on different factors within STATA V.14. We also plan to conduct a subgroup analysis on different elastography techniques, machines or other potential confounders.

\section{Sensitivity analysis}

To determine the robustness of the pooled results, we will conduct a sensitivity analysis by examining individual studies on estimated effects using STATA V.14.

\section{Data synthesis}

We plan to use Meta-Disc V.1.4 (Ramón y Cajal Hospital, Madrid, Spain) for threshold analysis and pooling analysis of diagnostic performance. The pooled sensitivity, pooled specificity, pooled positive likelihood ratio, pooled negative likelihood ratio and pooled diagnostic OR will all be synthesised by a random-effect model. The summary receiver operating characteristic (SROC) curve will be synthesised by a randomeffect model (DerSimonian-Laird model) if it is symmetrical, while the SROC curve will be synthesised by the MosesShapiro-Littenberg model if it is asymmetrical.

For data synthesis and statistical analysis on the pooled correlation coefficient and pooled OR, we will use STATA V.14 (StataCorp LP). A fixed-effect model will be used for analyses when heterogeneity is not present $(p>0.05)$. Otherwise, a random-effect model will be used.

\section{DISCUSSION}

Since Ophir J. first used elastography to image biological elasticity, ${ }^{15}$ the TE, ARFI and SWE techniques have been widely and well studied in adult liver fibrosis caused by viral hepatitis or non-alcoholic fatty liver disease. Although elastography techniques have limitations and confounding factors, they are all reliable and have good diagnostic performance based on current evidence. ${ }^{4}$ Quite a few studies have found that liver stiffness measured by TE, ARFI or SWE had good diagnostic performance in differentiating $\mathrm{BA}$ from other causes of neonatal cholestasis and assessing the severity of liver fibrosis. ${ }^{911} 1216$ Elastography techniques have been used to evaluate the degree of portal hypertension and predict liver-related events and other prognostic outcomes after HPE. ${ }^{791718}$

However, these studies vary in their results and even reach contrary conclusions. The study by Wang et al showed that supersonic SWE had a high area under the curve (AUC) of 0.997 for differentiating BA from infantile hepatitis syndrome, while Zhou et al only obtained an AUC of 0.790 using the same supersonic SWE technique. ${ }^{8} 19$ The liver or spleen stiffness measured by ARFI was reported to predict oesophageal varices after HPE. The study by Tomita H. et $a l$ found that liver stiffness measurements exhibited good predictive value for oesophageal varices, with a high AUC (0.90), while spleen stiffness measurements failed to predict oesophageal varices. ${ }^{20}$ In contrast, the research by Zhang et al demonstrated that spleen stiffness measured by ARFI predicted oesophageal varices with an AUC of 0.81 and that liver stiffness measurements failed to predict oesophageal varices. ${ }^{21}$ Many confounding factors, such as small sample sizes, may have led to contradictory conclusions.

This systematic review and meta-analysis will provide comprehensive evidence to evaluate the diagnostic performance and prognostic value of different elastography methods in patients with BA and after HPE.

\section{ETHICS AND DISSEMINATION}

As the study is a protocol of meta-analysis based on previously published literature, the ethical approval was not necessary according to the ethical committee of West China Hospital, Sichuan University. The results of this study will be published in a peer-reviewed journal.

\section{PATIENT AND PUBLIC INVOLVEMENT}

As the study is a protocol of meta-analysis based on previously published literature, the primary patient data will not be collected. Patient or public will not be involved in the study design, recruitment and data analysis.

Contributors HY, JL and YL conceived the study and drafted the manuscript. HY, $\mathrm{LD}, \mathrm{YL}$ and $\mathrm{JL}$ designed the search strategy and data extraction data set. JZ, YL and $J \mathrm{~L}$ formed the data synthesis and analysis plan. $J \mathrm{~L}$ and $\mathrm{YL}$ supervised this study and revised the manuscript. All authors have read and approved the publication of the protocol.

Funding This study was supported by grants from the China Postdoctoral Science Foundation (No. 2019M653422), the National Natural Science Foundation of China (No .82071940) and the Post-Doctor Research Project, West China Hospital, Sichuan University (№. 2018HXBH035).

Competing interests None declared.

Patient consent for publication Not required.

Provenance and peer review Not commissioned; externally peer reviewed.

Supplemental material This content has been supplied by the author(s). It has not been vetted by BMJ Publishing Group Limited (BMJ) and may not have been peer-reviewed. Any opinions or recommendations discussed are solely those of the author(s) and are not endorsed by BMJ. BMJ disclaims all liability and responsibility arising from any reliance placed on the content. Where the content includes any translated material, BMJ does not warrant the accuracy and reliability of the translations (including but not limited to local regulations, clinical guidelines, terminology, drug names and drug dosages), and is not responsible for any error and/or omissions arising from translation and adaptation or otherwise.

Open access This is an open access article distributed in accordance with the Creative Commons Attribution Non Commercial (CC BY-NC 4.0) license, which permits others to distribute, remix, adapt, build upon this work non-commercially, and license their derivative works on different terms, provided the original work is properly cited, appropriate credit is given, any changes made indicated, and the use is non-commercial. See: http://creativecommons.org/licenses/by-nc/4.0/.

\section{ORCID iDs}

Hualin Yan http://orcid.org/0000-0003-1338-1124

Juxian Liu http://orcid.org/0000-0002-5315-5482

\section{REFERENCES}

1 Bezerra JA, Wells RG, Mack CL, et al. Biliary atresia: clinical and research challenges for the twenty-first century. Hepatology 2018;68:1163-73.

2 Serinet M-O, Wildhaber BE, Broué P, et al. Impact of age at Kasai operation on its results in late childhood and adolescence: a rational basis for biliary atresia screening. Pediatrics 2009;123:1280-6.

3 Fawaz R, Baumann U, Ekong U, et al. Guideline for the evaluation of cholestatic jaundice in infants: joint recommendations of the North American Society for pediatric gastroenterology, hepatology, and 
nutrition and the European Society for pediatric gastroenterology, hepatology, and nutrition. J Pediatr Gastroenterol Nutr 2017;64:154-68.

4 Kennedy P, Wagner M, Castéra L, et al. Quantitative elastography methods in liver disease: current evidence and future directions. Radiology 2018;286:738-63.

5 Caruso M, Cuocolo R, Di Dato F, et al. Ultrasound, shear-wave elastography, and magnetic resonance imaging in native liver survivor patients with biliary atresia after Kasai portoenterostomy: correlation with medical outcome after treatment. Acta Radiol 2020;61:284185120902379

6 Chen S, Liao B, Zhong Z, et al. Supersonic shearwave elastography in the assessment of liver fibrosis for postoperative patients with biliary atresia. Sci Rep 2016;6:31057.

7 Yokoyama S, Ishigami M, Honda T, et al. Spleen stiffness by 2-D shear wave elastography is the most accurate predictor of high-risk esophagogastric varices in children with biliary atresia. Hepatol Res 2019;49:1162-8.

8 Zhou L-Y, Jiang H, Shan Q-Y, et al. Liver stiffness measurements with supersonic shear wave elastography in the diagnosis of biliary atresia: a comparative study with grey-scale us. Eur Radiol 2017;27:3474-84

9 Wu J-F, Lee C-S, Lin W-H, et al. Transient elastography is useful in diagnosing biliary atresia and predicting prognosis after hepatoportoenterostomy. Hepatology 2018;68:616-24.

10 Sintusek P, Siriporn N, Punpanich D, et al. Spleen and liver stiffness to detect esophageal varices in children with biliary atresia. $J$ Pediatr Gastroenterol Nutr 2019;69:411-5.

11 Chen Y, Zhao D, Gu S, et al. Three-Color risk stratification for improving the diagnostic accuracy for biliary atresia. Eur Radiol 2020;30:3852-61.

12 Gao F, Chen Y-Q, Fang J, et al. Acoustic radiation force impulse imaging for assessing liver fibrosis preoperatively in infants with biliary atresia: comparison with liver fibrosis biopsy pathology. $J$ Ultrasound Med 2017:36:1571-8.

13 Whiting PF, Rutjes AWS, Westwood ME, et al. QUADAS-2: a revised tool for the quality assessment of diagnostic accuracy studies. Ann Intern Med 2011;155:529-36.

14 Hayden JA, van der Windt DA, Cartwright JL, et al. Assessing bias in studies of prognostic factors. Ann Intern Med 2013;158:280-6.

15 Ophir J, Céspedes I, Ponnekanti H, et al. Elastography: a quantitative method for imaging the elasticity of biological tissues. Ultrason Imaging 1991:13:111-34.

16 Dillman JR, DiPaola FW, Smith SJ, et al. Prospective assessment of ultrasound shear wave elastography for discriminating biliary atresia from other causes of neonatal cholestasis. J Pediatr 2019;212:60-5.

17 Hanquinet S, Courvoisier DS, Rougemont A-L, et al. Acoustic radiation force impulse sonography in assessing children with biliary atresia for liver transplantation. Pediatr Radiol 2016;46:1011-6.

18 Hahn SM, Kim S, Park Kl, et al. Clinical benefit of liver stiffness measurement at 3 months after Kasai hepatoportoenterostomy to predict the liver related events in biliary atresia. PLoS One 2013;8:e80652

19 Wang X, Qian L, Jia L, et al. Utility of shear wave elastography for differentiating biliary atresia from infantile hepatitis syndrome. $J$ Ultrasound Med 2016;35:1475-9.

20 Tomita H, Ohkuma K, Masugi Y, et al. Diagnosing native liver fibrosis and esophageal varices using liver and spleen stiffness measurements in biliary atresia: a pilot study. Pediatr Radiol 2016;46:1409-17.

21 Zhang GY, Tang Y, Niu NN, et al. [Clinical value of acoustic radiation force impulse technique to predict esophageal and gastric varices in patients with biliary atresia]. Zhonghua Yi Xue Za Zhi 2017;97:525-8. 